\title{
Magnetic Resonant Image and Ultrasound with Doppler of Soft- Tissue Lesion
}

\author{
Ahmed Youssef, Ahmed Torky, Amal Gamal
}

Department of Radiodiagnosis, Benha faculty of medicine, Benha University, Egypt.

Correspondence to: Amal Gamal, Department of Radiodiagnosis, Benha faculty of medicine, Benha University, Egypt.

Email:

amalelkoly89@gmail.com

Received: 15 December 2020

Accepted: 9 February 2021

\begin{abstract}
Background: Imaging of soft tissue tumors requires a multimodality approach. Ultrasound (US) or MRI is chosen according to the clinical characteristics, location of the soft tissue tumor, and patient. The aim of the present study was to establish new diagnostic criteria and improve the diagnostic efficacy of MRI and US with Doppler in assessment of soft-tissue lesions. Patients and methods: This cross-sectional study was conducted at Radiology Department; Benha University during the period from February 2018 to September 2019. Inclusion criteria: Patients who suffer from soft tissue lesions either superficial or deep in both sex and any age group. Ultrasound can detect fine details of small lesions. MR imaging can evaluate tumour staging, neur191ovascular involvement, tumour necrosis and preoperative planning. Results: The study included 60 patients with soft-tissue lesion (19 females and 41 males), their ages ranged from 5 to 70 years with the mean of age $30.6 \pm 15.8$. The most common lesion
\end{abstract} diagnosed among the studied group was hemangioma $(21.66 \%)$ and picture of ganglion (13.33\%) followed by moderate degree of osteoarthritis (11.66\%). Conclusion: Magnetic Resonance Imaging (MRI) is a well-established imaging tool for the detection and local staging of soft-tissue tumors. MR imaging exhibited different advantages like determining the origin of these lesion in defining their extent and relation to adjacent structures, assessing operability by identifying osseous, neurovascular bundles and joint space involvement by soft tissue tumors. This would help provide a non-invasive diagnosis of such lesions, consequently improving patient management.

Keywords: MRI; US; Doppler; Soft-Tissue; Lesion 


\section{Introduction}

Soft tissue lesions refer to damage inflicted on the connective tissue of the body. Soft tissue lesions strike fear in many pathologists as they are uncommon and may be difficult to diagnose (1). Accurate assessment of the location, extent, borders, and signal intensity is paramount for the characterization of softtissue lesions (2).

Soft tissue malignancies are an uncommon heterogeneous group of mesenchymal lesions. They account for $1 \%$ of adult malignant tumors and are estimated to represent about $1 \%$ of all malignancy (3).

Imaging of soft tissue tumour requires a multimodality approach, with no single imaging modality being ideal for every tumour (4).

Primary ultrasound (US) or MRI is chosen according to the clinical characteristics, location of the soft tissue tumor, and patient "concern". MRI is the modality of choice for diagnostic and local staging of soft tissue tumors, but US may be enough for simple, superficial, non-growing benign lesions. Computed tomography (CT) is indicated to better define the osseous and matrix architecture and in patients with contraindications to MRI (5).
MRI is fast outpacing any other modality for in vivo viewing of soft tissues in the human body without the need to resort to any invasive procedures. MRI scan does not use ionizing radiation. MRI offers the best resolution of tissues of low inherent contrast. (6) .

MRI lesion characterization includes assessment of signal intensity, size, morphology, location, and relationship to adjacent structures, and multiplicity (including other lesions on the field of view, eg, lymph nodes, skip metastases) (5).

The ultrasonography is a non-invasive, inexpensive and painless imaging method. Reliability of power Doppler technology is an issue and requires standardization of the technique .Differential diagnosis can be made easily by Doppler US (7). US-guided aspiration can be tested to confirm the diagnosis (8).

Preoperative diagnosis of most soft-tissue lesions is possible by combining the USbased sarcoma screening (USS) score with characteristic clinical and MRI findings (7).

The aim of the present study was to establish new diagnostic criteria and improve the 
diagnostic efficacy of MRI and US with

Doppler in assessment of soft-tissue lesions.

\section{Patients and methods}

This cross-sectional study was conducted at Radiology Department; Banha University during the period from February 2018 to September 2019. The study included 60 patients with soft-tissue lesion. Informed consent had been taken from all the participants or their relatives in this study. This study was approved by our Institutional Review Board (IRB).

Patient inclusion criteria:

Patients who suffer from soft tissue lesions either superficial or deep in both sex and of any age group, are included in the study. Ultrasound can detect fine details of small lesions. MR imaging can evaluate tumour staging, neurovascular involvement, tumour necrosis and preoperative planning.

Patient exclusion criteria:

1. Patient having history of claustrophobia.

2. Patient having history of metallic implants insertion, cardiac pacemakers and metallic foreign body in-situ.

3. Patients unwilling to complete study. tients were subjected to:

Detalled history taking:

1. Full history was taken including the following: Personal history: age and sex of patient.

2. Physical examination: Include both general and local examination done by the clinician.

3. Radiological evaluation

A) Sonographic analysis of lesions

B) Magnetic resonance imaging (MRI)

Statistical Analysis

Data were analyzed using Statistical Program for Social Science (SPSS) version 23 and MedCalc version 15.4. Quantitative data were expressed as mean \pm standard deviation (SD). Qualitative data were expressed as frequency and percentage.

\section{Results}

Patient's age ranged between 5-70 years with mean of 30.6 years. The majority of them $(48.33 \%)$ were in 21-35 year's group followed by $26.66 \%$ in 5-20 year's group. It was also noticed that $68.33 \%$ were males compared to $31.66 \%$ females. Table 1 
Table 2, shows that the most common MRI characteristic of lesion among the studied group was lobulated mass, low SI in T1, high T2 and STAIR, small foci of signal void, normal surrounding structure $(21.66 \%)$ and abnormal signal intensity of low T1, high T2 normal ACL, PCL abnormal signal in meniscus high in T2 moderate degree (11.66\%).

Table 3, shows that the most common US with doppler diagnosis of lesion among the studied group was soft tissue mass hemangioma $(21.66 \%)$ and picture of ganglion $(13.33 \%)$ followed by moderate degree of osteoarthritis with bulge in lateral meniscus $(11.66 \%)$.

Case 1: Figure 1

Fibroelastoma; female patient 48 yrs presented with a non-painful swelling at the left upper back. Ultrasound examination show infrascapular well defined multilayered soft tissue lesion measure about $24 \mathrm{~mm}$ x 21 $\mathrm{mm}$ with no color on doppler; by using MRI examination the lesion is isointense to the muscles in $\mathrm{T} 1$ and $\mathrm{T} 2$ with no invasion, collection or sinus connection.... feature of fibroelastoma dorsi.

\section{Case 2: Figure 2}

194 ion: female patient 33yrs presented wıuı non-painful swelling at the right hand. By ultrasound exam we can see cystic lobulated soft tissue lesion related to the extensor carpiradialis tendon measure about $19.5 \mathrm{mmx} 10.6 \mathrm{~mm}$ with no color on doppler. MRI examination show cystic mass at the level of metacarporadial joint with signal intensity low in T1, high in $\mathrm{T} 2$ with intact tendon course giving the picture of ganglion formation

\section{Case 3: Figure 3}

Giant cell tumour: female patient 24 yrs presented with non-painful soft tissue lesion at lateral side of biggest toe of left foot, on ultrasound examination we can soft tissue lesion related to the extensor tendon of big toe measure about $16 \times 5 \mathrm{~mm}$ with mild color on doppler. On MRI with contrast scanning with fat suppression sequence, the lesion is isointense in $\mathrm{T} 1$, hyper intense in $\mathrm{T} 2$ with moderate enhancement, no surrounding bones destruction..... most probably giant cell tumour. 
Table (1): Demographic data of the studied group:

\begin{tabular}{lll}
\hline Variable & Studied group $(\mathrm{n}=60)$ \\
\hline Age: & \multicolumn{2}{l}{$30.6 \pm 15.8$} \\
Mean \pm SD & $5-70$ & \\
Range & No & $\%$ \\
& & \\
Age: (years) & 16 & 26.66 \\
$\mathbf{5 - 2 0}$ & 29 & 48.33 \\
$\mathbf{2 1 - 3 5}$ & 7 & 11.6 \\
$\mathbf{3 6 - 5 0}$ & 6 & 10 \\
$\mathbf{5 1 - 6 5}$ & 2 & 3.33 \\
$\mathbf{6 6 - 8 0}$ & No & $\%$ \\
& & \\
Sex: & 41 & 68.33 \\
Male & 19 & 31.66 \\
Female & &
\end{tabular}

Table (2): MRI characteristic of lesion among the studied group:

Variable

studied group $=60$

Characteristic:

No. \%

Intramuscular abnormal SI hetrogenous low T1,high T2 post contrast enhacment 'no invasion of surround structures

$1 \quad 1.66$

Lobulated mass, low SI in T1, high T2 and STAIR, small foci of signal void, normal surrounding structure

$13 \quad 21.66$

Thick periosteal reaction and surrounding soft tissue component of low signal in T1,T2, mixed high signal in STAIR. $1 \quad 1.66$ low fibula , talus , calcenous show low T1 ,T2, high STAIR, no associated soft tissue mass and muscle affection

Lobulated mass, low SI in T1, high T2 and STAIR, small foci of signal void, normal surrounding structure 
Left infrascapular soft tissue mass isointense in pattern in T1,T2 no collection, no soft tissue invasion, no joint $1 \quad 1.66$ effusion

Cystic mass, low T1, high T2, normal tendon course and surrounding structure

195 ic mass, low T1, high T2, normal tendon course and surrounding structure, fine internal septations

ivnutiple well-defined cystic lesions. low T1, high T2 normal surrounding structure

Well defined subcutaneous mass, heterogenous high signal intensity in T1, T2 and suppressed in STAIR with nodularity 2 and thick septation, no calcification

Cystic mass with intact tendon course, another tiny similar lesion of low T1, high T2 normal surrounding structure

Well defined lobulated abnormal signal intensity of low heterogenous internal signal intensity $\quad$.... picture suggest 1 pseudoanurysm with internal thrombosis. Normal surrounding bone ,muscle ,ligment

Right perianal fistula extend from external opening through ischeoanal fossa , intetrupt internal sphincter .Small 1 collection at ischeo rectal fossa normal levator ani

Well defined hemogenous signal in $\mathrm{T} 2$ and proton denisty and decrease signal in $\mathrm{T} 1$ typically signal of subcutaneus fluid. 2

Lobulated mass, low SI in T1, high T2 and STAIR, small foci of signal void, normal surrounding structure, bursa show $4 \quad 6.66$ low SI in T1, high T2

Localized subcutanous soft tissue mass isointense in $\mathrm{T} 1$, hyperintense in T2 with moderate enhacement with hypintense 1 capsule surrounding no bone destruction surrounding

Abnormal signal intensity in of isointense in T1, dark signal in T2 agree with early subacute blood collection normal 2 surrounding structure

MRI confirm the nature of being lymphatic malfomation more than venous category however percutanous injection of 1 contrast on basis of intervention is final dignostic test

Abnormal area of low signal intensity, heterogenous in $\mathbf{T 1}$,higher in T2 surrounded by enhacned rim surrounded by low $3 \quad 5.00$ signal of edema normal surrounding structure

Abnormal signal intensity of low T1, high T2 normal ACL , PCL abnormal signal in meniscus high in T2 moderate $7 \quad 11.66$ degree 


\section{Characteristic:}

Soft tissue mass with malignant character

Soft tissue mass hemangioma

Soft tissue elevation of periosteum

Diffuse venous malformation

Post traumatic nerve neuroma

Fibroelastoma

Picture of ganglion

Picture of recurrent ranula

Fat containing soft tissue mass

$3 \quad 5.00$

\section{Complicated lipoma}

Cystic mass with internal septation

Ulnar artery partial thrombosis pseudoaneurysm for angiographic study

1.66

Collection for MRI assessment

Lateral mallular bursa effusion

Severe wrist bursitis with effusion

$4 \quad 6.66$

Mostly giant cell tumor of tendon

Heamatoma

Multicystic lesion

Localized collection 

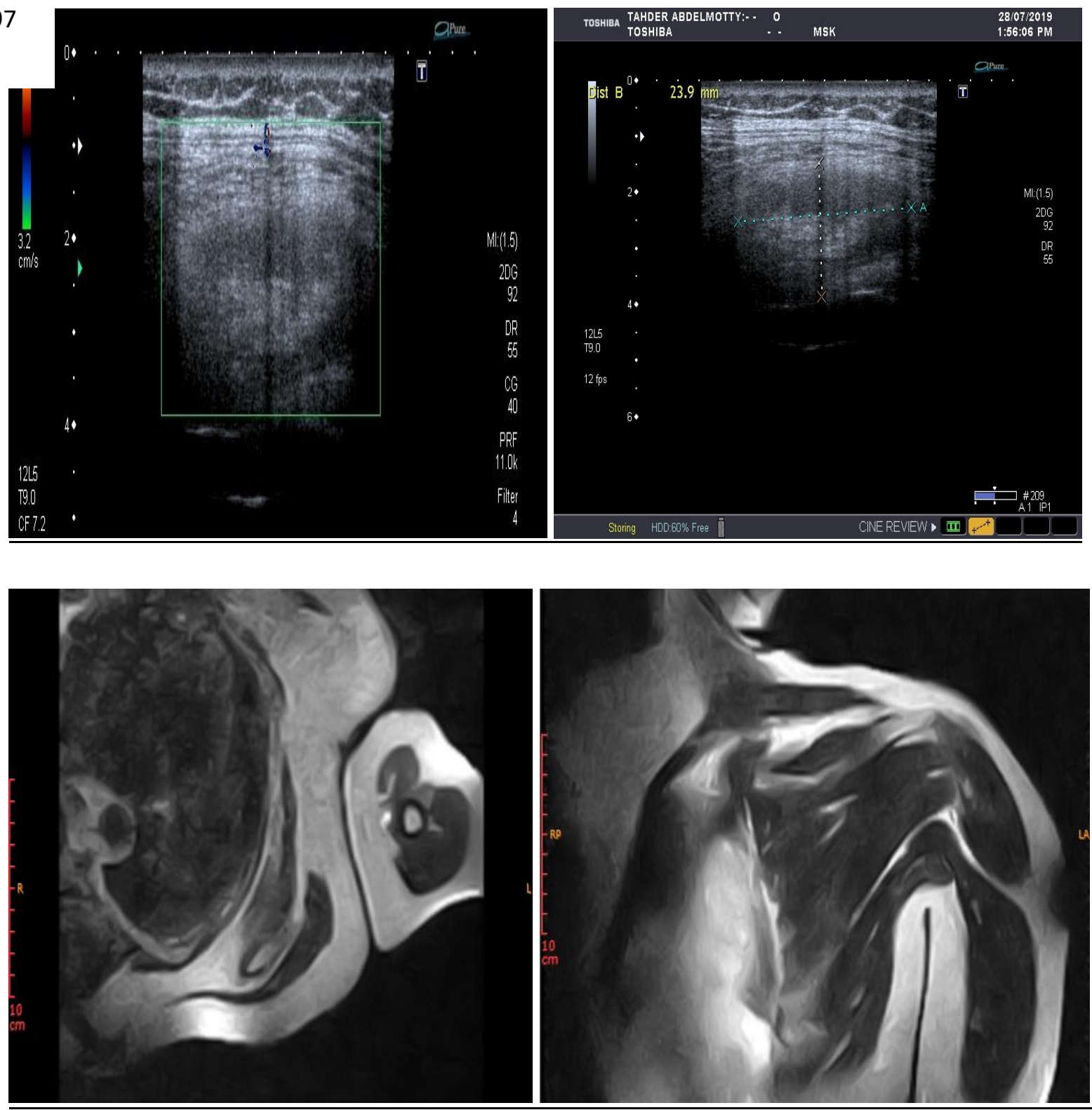

Figure 1: Axial T2

Cronal T 

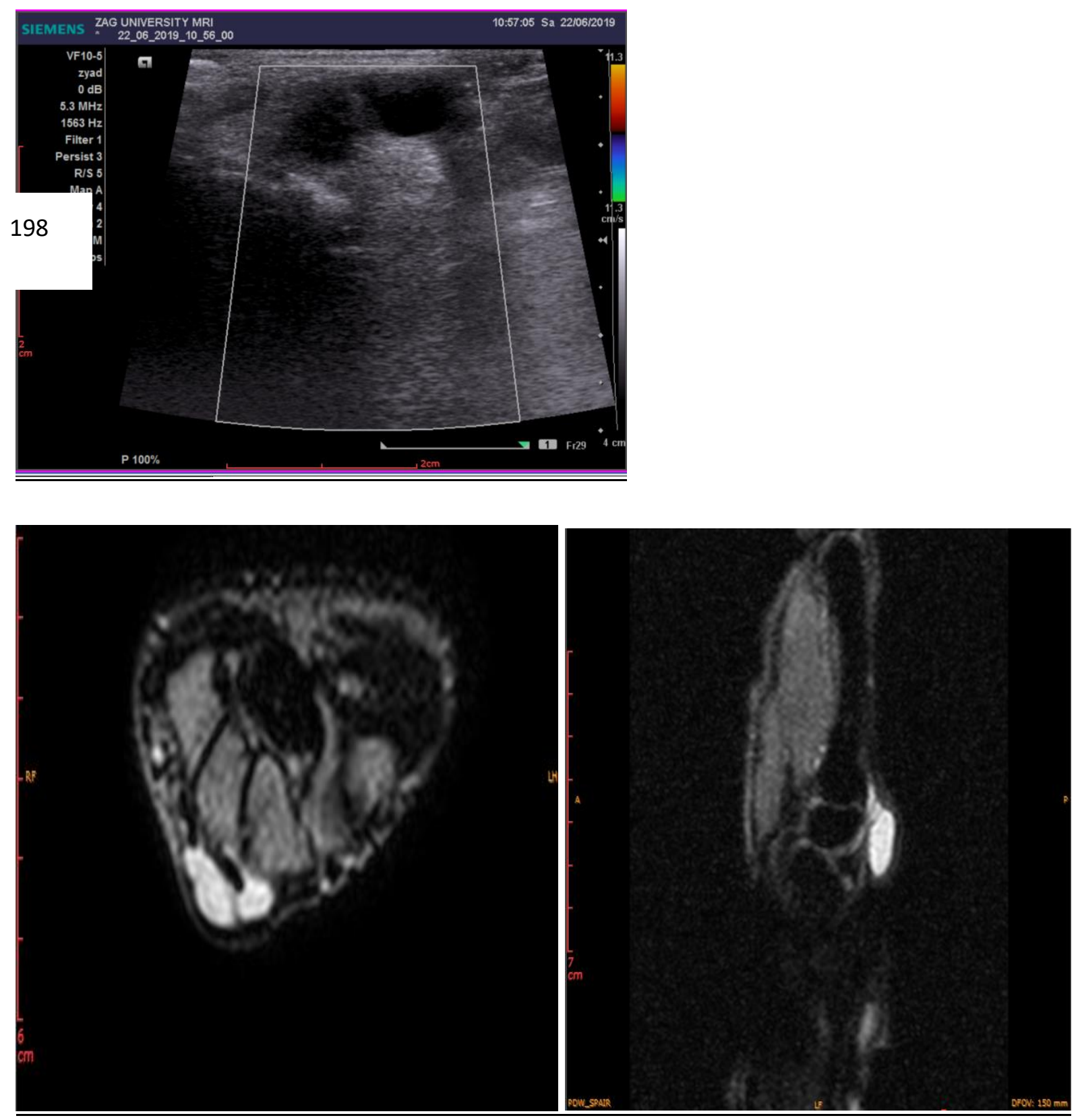

Figure 2: Stair axial

T2 saggital 

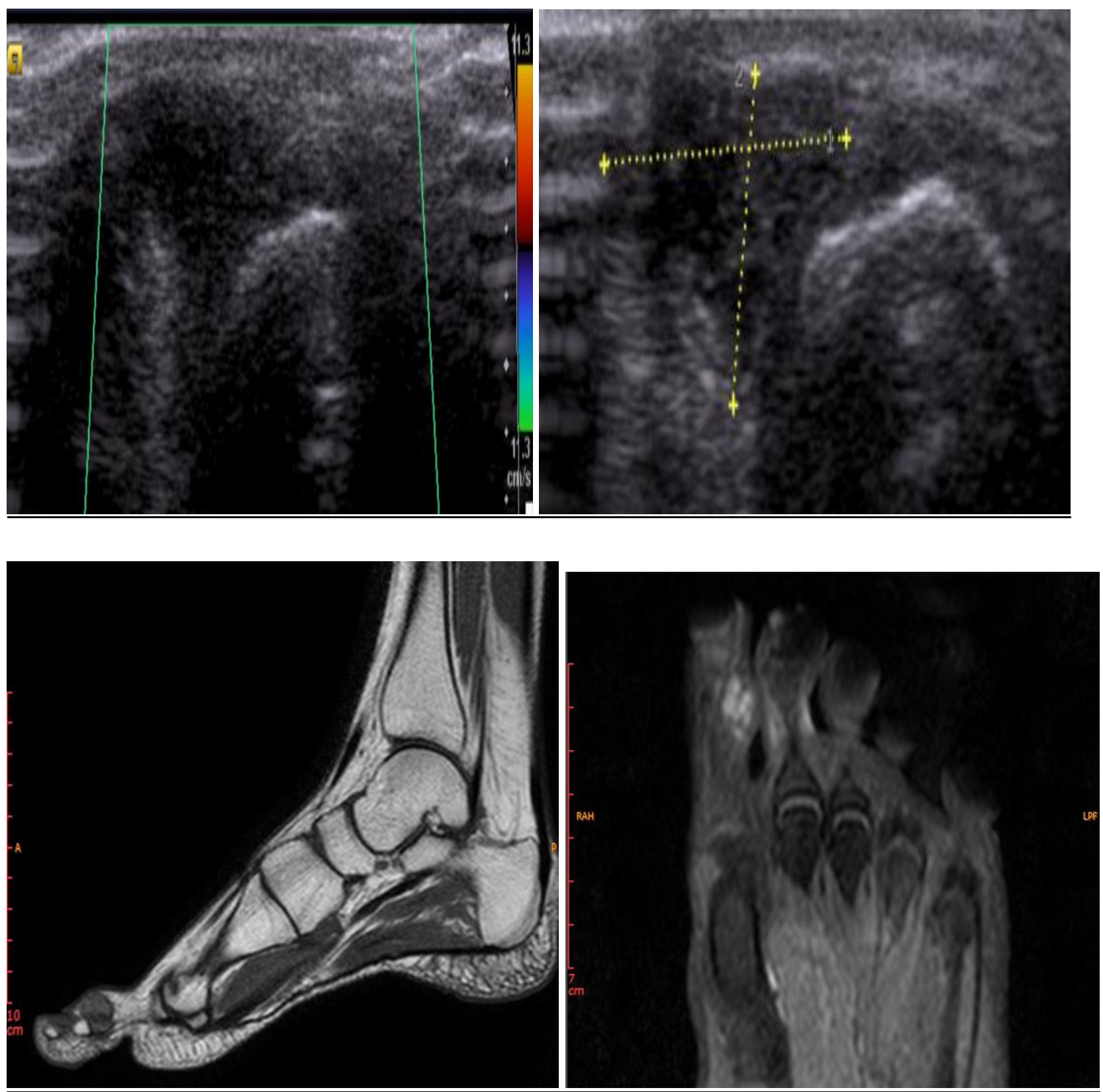

Figure 3: Stair saggital

$\mathrm{T} 2$ cronal

and 35 -years while $26.66 \%$ were between 5

\section{Discussion} and 20 years. the mean age is $30.6 \pm 15.8$

The present study included sixty patients, their ages ranged between 5-70 years the majority of them (48.33\%) were between 21-

years. The majority of our study population was males $68.33 \%$ versus $31.66 \%$ females. 
Different patients' characteristics were found in the another study, (9) which investigated the diagnostic accuracy of colour doppler ultrasound in 180 patients (87 males, 93 female) ranged from 1 to 91 years (mean $58.1 \pm 20.0$ years) with soft tissue tumours.

In the present study hemangioma was detected in $21.66 \%$ of studied group. By US with Doppler, it appears as ill-defined hyperechoic mass with multiple hypoechoic tubular structure. By MRI, it appears as lobulated mass, low SI in T1, high T2 and STAIR, small foci of signal void, normal surrounding structure .Hemangiomas may contain a variable amount of adipose tissue interspersed between the abnormal vessels. However, its frequently typical appearance on MR images, owing to the presence of the high SI of slow-flowing blood within serpentine or tubular structures, allows the correct diagnosis to be made (10).

In the present study, elastofibroma dorsi was found in $1.66 \%$ of studied group. By US with Doppler, it appears as soft tissue lesion well defined, mixed echogenicity measure deep to serratus anterior and latissimus dorsi. By MRI, it appears as infra-scapular soft tissue mass, iso-dense intense pattern in $\mathrm{T} 1, \mathrm{~T} 2$ no collection, no soft tissue invasion, no joint effusion.
In the current study, sarcomatous lesion was found in $1.66 \%$ of studied group. By US with Doppler, it appears as lobulated soft tissue mass with daughters with multiple area of nerrosis with minimal color with Doppler. 201 MRI, it appears as intramuscular uunurmal SI heterogeneous low T1, high T2 post contrast enhancement measure $27 * 13 \mathrm{~cm}$ in hamstring, anterior extensor muscle of thigh no invasion in femur.

In the current study, lipoma was found in 5\% of studied group. By US with Doppler, it appears as fat containing soft tissue mass. By MRI, it appears as well defined intramuscular soft tissue mass high SI, T1, T2.

US with CDUS is ideally performed with a variable frequency probe in which the upper range of the frequencies $(15-18 \mathrm{mHz})$ are used for the evaluation of the skin layers (epidermis-dermis) and the frequencies lower than $13 \mathrm{mHz}$ are used for the deeper tissues. High and ultrahigh resolution $(15 \mathrm{mHz}$ and $20 \mathrm{mHz}$ and higher, respectively) has a definite better diagnostic accuracy for evaluation of lesions superficial to the investing fascia and at the skin (11).

Addition of US to clinical examination improved correctness of the diagnosis to 97 $\%$ as calculated by Wortsman in a 
retrospective study in 4338 US examinations (11).

In the current study, ganglion was found in $13.33 \%$ of studied group. By US with Doppler, it appears as cystic soft tissue mass. By MRI, it appears as cystic mass, low T1, high T2, normal tendon course and surrounding structure.

Ganglia are benign cystic lesions that arise from the joint capsule or tendon sheath, most commonly from the limb joints, with rare occurrence at the spine. They contain mucinous fluid, which is highly viscous, proteinaceous material, rich in hyaluronic acid, glycosamine, albumin, and globulin, but their wall consists of a (discontinuous) layer of flattened pseudo synovial cells, surrounded by dense fibrous connective tissue (pseudo capsule) (12).

The ganglion is a cystic, glue-like mass containing fluid and lined with collagen within the epineurium that may cause pain and motor dysfunction due to compression (13). These are non-neoplastic cysts caused by the accumulation of thick mucinous fluid through a connecting branch with a neighboring joint with growth within the epineurium of peripheral nerves (14). US shows a spindle-shaped anechoic soft tissue structure within or abutting the nerve course (13).

In the current study, ulnar artery partial thrombosis pseudo aneurysm was found in $1.66 \%$ of studied group. By US with Doppler, it appears as ulnar artery with 202 thrombosis forming pseudo aneurysm. I, it appears as well defined lobulated abnormal signal intensity of low heterogeneous internal signal intensity picture suggests pseudo aneurysm with internal thrombosis with normal surrounding bone, muscle and ligament.

Pseudo aneurysms appear as hypoechoic or anechoic lesions with increased throughtransmission. Internal echogenicity, representing thrombus and septations, may be seen. US has unique advantages in depicting the distinguishing imaging features of pseudo aneurysms from hematoma. High spatial resolution allows visualization of their relationship to adjacent arteries, and the use of colour Doppler US reveals the vascular nature of these lesions (15)

Soft tissue tumour entirely surrounds and obliterates a major artery, an aneurysm or a pseudo aneurysm should be considered. Peripheral arterial aneurysms are most commonly found in the popliteal and ulnar 
artery, often in association with widespread atherosclerotic disease (16).

In the current study, lateral malleolar bursa effusion was found in $3.33 \%$ of studied group. By US with Doppler, it appears as compartment swelling of ankle joint containing anechoic fluid in subcutaneous lateral malleolar bursa. By MRI, it appears as well-defined homogenous signal in T2 and proton density and decrease signal in $\mathrm{T} 1$ typically signal of subcutaneous fluid.

A normal bursa is not visualized on ultrasound or is seen only as a thin hypoechoic space or sac in a typical anatomical location. When a bursa is distended, it appears as a hypoechoic structure with well-defined margins and contents of variable echogenicity. In a simple bursitis, there may be just anechoic fluid, with or without septa. In chronic bursitis due to impingement or overuse, more frequently there is bursal wall thickening, with internal debris of variable echogenicity (17).

In the current study, soft tissue mass mostly giant cell tumour was found in $1.66 \%$ of studied group. By US with Doppler, it appears as soft tissue mass related to tendon with mild color in Doppler signal. By MRI, it appears as localized subcutaneous soft tissue mass isointense in T1, hyper intense in $\mathrm{T} 2$ with moderate enhancement with hypo intense capsule surrounding no bone destruction surrounding. Some lesions may not contain enough hemosiderin to be $\mathrm{T} 1$ and T2 hypo intense or to cause a blooming artifact on gradient-echoimages (18).

In the current study, early subacute hematoma was found in $3.33 \%$ of studied group. By US with Doppler, hematoma seen in middle compartment of quadriceps muscle with moderate to marked flow on color Doppler. By MRI, it appears as abnormal signal intensity of isointense in $\mathrm{T} 1$, dark signal in T2 agree with early subacute blood collection normal surrounding structure.

The ultrasound appearance of hematomas is variable in time. Acute hematomas are hyperechoic and they become more hypoechoic with aging. They may have welldefined or irregular margins. Dynamic evaluation with muscle contraction is valuable for assessing disruption of muscle architecture. The MR imaging appearance of muscular hematomas reflects the pathophysiology of forming hemoglobin breakdown products, which are the main constituents of a hemorrhagic collection (18). A hematoma in the early subacute stage is characterized by the presence of intracellular 
methemoglobin. This produces high signal intensity on T1-weighted images, often visualized as a high-intensity peripheral rim. Susceptibility effects persist on T2-weighted images. When loss of cell compartmentalization occurs in late subacute hematomas, extracellular methemoglobin results in $\mathrm{Tl}$ shortening. On $\mathrm{T} 2$-weighted images, the hematoma may be outlined by an area of high signal intensity. Diffuse edema is also present within the muscle in acute and subacute hematomas. Finally, hemosiderin in a chronic hematoma also produces susceptibility effects on T2-weighted images. This results in low signal intensity on T1weighted images and particularly on T2weighted images. Blooming artifact is seen when using gradient echo imaging. Furthermore, this phenomenon is accelerated at the periphery of the collection, resulting in a peripheral hypo intense rim, whereas the central portion of the hematoma may remain hyper intense (19)

In the current study, Lymphatic malformation was found in $1.66 \%$ of studied group. By US with Doppler, it appears as multi-cystic lesion with no color on Doppler (no rasterization flow) with internal septa with anechoic content. By MRI, it confirms the nature of being lymphatic malformation more than venous category however percutaneous injection of contrast on basis of intervention is final diagnostic test sclerotherapy under imaging is the treatment.

Kabilan et al. (20) found that lymphatic malformation by transverse gray scale ultrasound shows multiloculated cystic lesion in the right side of neck. The cystic spaces are well seen. The cystic spaces are not ressible by the ultrasound probe. On 204 : doppler, the lesion does not show any mitenal vascularity. Coronal T2W sequence shows an ill-defined, multiloculated heterogeneously hyper intense lesion with multiple thin hypo intense internal septations. On coronal $\mathrm{T} 1 \mathrm{~W}$ sequence the lesion is iso to hyper intense. On coronal T1 fat suppressed post contrast sequence, the lesion does not show any contrast enhancement.

\section{Conclusion}

An extensive review of ultrasound and MRI appearances of soft tissue lesions focusing on uncommon and atypical ones would help radiologists to expand their knowledge of the same and accurately diagnose such lesions. Magnetic Resonance Imaging (MRI) is a well-established imaging tool for the detection and local staging of soft-tissue tumors. MR imaging exhibited different advantages like determining the origin of these lesion in defining their extent and 
relation to adjacent structures, assessing operability by identifying osseous, neurovascular bundles and joint space involvement by soft tissue tumors. This would help provide a non-invasive diagnosis of such lesions, consequently improving patient management. This would be expected to significantly reduce unnecessary biopsies in benign lesions and allow early diagnostic interventions and management in malignant or aggressive lesions, thereby greatly improving prognosis and patient outcomes.

\section{References}

1. Beaman FD, Jelinek JS, Priebat DA. Current imaging and therapy of malignant soft tissue tumors and tumor-like lesions. In: Seminars in musculoskeletal radiology. Thieme Medical Publishers; 2013. p. 168-76.

2. Chhabra A, Soldatos T. Soft-tissue lesions: when can we exclude sarcoma? Am J Roentgenol. 2012;199(6):1345-57.

3. Afonso PD, Kosinski AS, Spritzer CE. Following unenhanced MRI assessment for local recurrence after surgical resection of mesenchymal soft tissue tumors, do additional gadolinium-enhanced images change reader confidence or diagnosis? Eur J Radiol. 2013;82(5):806-13.

4. Peterson JJ. Current developments and recent advances in musculoskeletal tumor imaging. In: Seminars in musculoskeletal radiology. Thieme Medical Publishers; 2013. p. 99-100.
5. Afonso PD, Mascarenhas V V. Imaging techniques for the diagnosis of soft tissue tumors. Reports Med Imaging. 2015;8:63-70.

6. Shah N, Bansal N, Logani A. Recent advances in imaging technologies in dentistry. World $\mathbf{J}$ Radiol. 2014;6(10):794.

7. Nagano S, Yahiro Y, Yokouchi M, Setoguchi T, Ishidou Y, Sasaki H, et al. Doppler ultrasound for diagnosis of soft tissue sarcoma: efficacy of ultrasound-based screening score. Radiol Oncol. $3015 \cdot 40 / 7) \cdot 135-40$.

205

'prak H, Kiliç E, Serter A, Kocakoç E, S. Ultrasound and Doppler US in evaluation of superficial soft-tissue lesions. J Clin Imaging Sci. 2014;4.

9. Oebisu N, Hoshi M, Ieguchi M, Takada J, Iwai T, Ohsawa M, et al. Contrast-enhanced color Doppler ultrasonography increases diagnostic accuracy for soft tissue tumors. Oncol Rep. 2014;32(4):1654-60.

10. Battaglia M, Vanel D, Pollastri P, Balladelli A, Alberghini M, Staals EL, et al. Imaging patterns in elastofibroma dorsi. Eur J Radiol. 2009;72(1):16-21.

11. Wortsman X, Wortsman J. Clinical usefulness of variable-frequency ultrasound in localized lesions of the skin. J Am Acad Dermatol. 2010;62(2):247-56.

12. Nikolopoulos I, Krinas G, Kipriadis D, Ilias A, Giannakopoulos A, Kalos S. Large infrapatellar ganglionic cyst of the knee fat pad: a case report and review of the literature. J Med Case Rep. 2011;5(1):17. 
13. Lee D-Y, Hwang S-C, Jeong S-T, Nam D-C, Park J-S, Lee J-H, et al. The value of diagnostic ultrasonography in the assessment of a glomus tumor of the subcutaneous layer of the forearm mimicking a hemangioma: a case report. J Med Case Rep. 2015;9(1):1-6.

14. Spinner RJ, Harish S, Amrami KK. An historical perspective on ulnar intraneural ganglion cysts and their joint origins. SAGE Publications Sage CA: Los Angeles, CA; 2014.

15. Carra BJ, Bui-Mansfield LT, O'Brien SD, Chen DC. Sonography of musculoskeletal soft-tissue masses: techniques, pearls, and pitfalls. Am J Roentgenol. 2014;202(6):1281-90.

16. Vanhoenacker FM, Parizel PM, Gielen JLMA. Imaging of soft tissue tumors. Springer Science \& Business Media; 2006.
17. Teefey SA, Dahiya N, Middleton WD, Gelberman RH, Boyer MI. Ganglia of the hand and wrist: a sonographic analysis. Am J Roentgenol. 2008;191(3):716-20.

18. Narváez JA, Narváez J, Aguilera C, De Lama E, Portabella F. MR imaging of synovial tumors and tumor-like lesions. Eur Radiol. 2001;11(12):2549-60.

19. Bush $\mathrm{CH}$. The magnetic resonance imaging of musculoskeletal hemorrhage. Skeletal Radiol. 2000;29(1):1-9.

20. Kabilan K, Sethy A, Krishnan V, Pansari S. The hard part of soft tissue lesions: Diagnosis by ultrasound and magnetic resonance imaging. In European Congress of Radiology-ECR 2019; 2019.

To cite this article: Ahmed Youssef, Ahmed Torky, Amal Gamal. Magnetic Resonant Image and Ultrasound with Doppler of Soft-Tissue Lesion. BMFJ 2021;38 (Radiology): 191-206 DOI: 10.21608 bmfj.2020.52360.1351 